\title{
The Entanglement of a Two-Atomic System in the Presence of a Silver Nanosphere
}

\author{
Roya Mombeiny Godazhdar ${ }^{a}$, Ehsan Amooghorban ${ }^{\mathrm{a}, \mathrm{b}, \mathrm{c}}$, and Ali Mahdifar ${ }^{\mathrm{d}, \mathrm{e}}$ \\ ${ }^{a}$ Department of Physics, Faculty of Basic Sciences, Shahrekord University, Shahrekord, Iran \\ ${ }^{b}$ Photonics Research Group, Shahrekord University, Shahrekord, Iran \\ ${ }^{c}$ Nanotechnology Research Center, Shahrekord University, Shahrekord, Iran \\ ${ }^{d}$ Department of Physics, Faculty of Science, University of Isfahan, Hezar Jerib, Isfahan, Iran \\ ${ }^{\text {e}}$ Quantum Optics Group, Department of Physics, Faculty of Science, University of Isfahan, \\ Hezar Jerib, Isfahan, Iran
}

*Corresponding Author Email: roya.m.g72@gmail.com

\begin{abstract}
Regular paper: Received: Jul. 12, 2019, Revised: Nov. 15, 2019, Accepted: Jan. 16, 2020, Available Online: June. 30, 2020, DOI: 10.29252/ijop.14.1.99
\end{abstract}

\begin{abstract}
In this paper, we study the entanglement of two-level atoms near a spherical silver nanoparticle. By employing the Von Neumann equation and utilizing of the electromagnetic Green's tensor associated with a dispersive and dissipative dielectric sphere, the decay rates and the Lamb shift of the atomic system are obtained. Then, by using the concurrence measure, we calculate the degree of entanglement of the atomic system. We observe that the decay rates severely increase near the excitation frequency of the localized plasmon-polariton, while the concurrence value is nearly zero.
\end{abstract}

KEYWORDS: Decay rates, Localized surface plasmon-polariton, Entanglement, Concurrence.

\section{INTRODUCTION}

Quantum entanglement is one of the most prominent features of quantum mechanics, which has attracted much attention in various physical fields, such as quantum optics, quantum information and quantum computation [1]. In fact, entanglement is a direct consequence of the superposition principle in quantum mechanics that does not have a classical counterpart. The entanglement makes it impossible to characterize the state of a composite system completely in terms of product states of constituent subsystems.

In general, a quantum state of a bipartite system is separable, if its density operator can be written as $\rho_{A B}=\sum_{i} P^{i} \rho_{A}^{i} \otimes \rho_{B}^{i}$ in which $P^{i} \geq 0 \cdot \sum_{i} P^{i}=1$ and $\rho_{A(B)}^{i}$ are the density of the A (B) sub-system operators [2]. Due to the inevitable interactions between atomic subsystems and the environment, a pure state will be driven to a mixed state under the environment induced decoherence and dissipation. Therefore, it is very difficult to say whether a system with an arbitrary state is separable. Hence, we need an appropriate measure to determine the degree of entanglement. For mixed states with the Hilbert space of dimension $2 \times 2$, the concurrence and the negativity are appropriate measures of the entanglement [3].

In this paper, we investigate the entanglement dynamic between two identical two-level atoms located near a silver nanosphere (SNS) by utilizing the concurrence. The two-atomic 
system is one of the simplest composite systems, which can show the fundamental features of quantum mechanics such as entanglement. The metallic nanosphere, however, can exhibit a localized surface plasmon (LSP) resonance at the frequency of the atomic transition. Due to the enhancement of the local density of states (LDOS) approximate to the SNS, we expect that the decay rates and subsequently the concurrence would be significantly different at frequencies that two atoms resonantly coupled to the LSP.

\section{BASIC EQUATIONS}

We consider a two identical two-level atoms $A_{1}$ and $A_{2}$ with identical transition frequencies, $\omega_{0}$, at distance $r_{1}=-r_{2}=r \hat{z}$, from the center of a SNS with radius $\alpha$. The origin of the coordinate system coincides with the center of the SNS and the two identical atoms (TIA) are placed symmetrically outside the SNS on the $\mathrm{z}$ axis of the coordinate system. To describe the quantum features of the TIASNS interactions, a quantization scheme of the electromagnetic field in the presence of absorbing medium is needed. The scheme was developed based on two approaches: canonical [4-10] and phenomenological [11-13] methods. In the present paper, we use the rigorous canonical formalism. Based on this microscopic approach, the quantized electric field is given by $[8,10,14]$

$$
\begin{aligned}
\mathbf{E}(\mathbf{r}, \omega)= & i \sqrt{\frac{\hbar}{\pi \varepsilon_{0}}} \frac{\omega^{2}}{c^{2}} \int d^{3} \mathrm{r} \sqrt{\varepsilon_{I}\left(\mathrm{r}^{\prime}, \omega\right)} \overline{\overline{\mathbf{G}}}\left(\mathbf{r}, \mathbf{r}^{\prime}, \omega\right) \\
& \cdot \mathrm{f}\left(\mathbf{r}^{\prime}, \omega\right) \mathrm{e}^{-i \omega t}+\text { H.C. }
\end{aligned}
$$

where H.C. denotes Hermitian conjugate, $\varepsilon_{0}$ is the vacuum permittivity, $c$ is the vacuum speed of light, $\varepsilon_{I}$ is the imaginary part of the permittivity function, $\varepsilon$. Here, $\mathbf{f}\left(\mathbf{r}^{\prime}, \omega\right)$ is the annihilation operator of the electric excitation of the system satisfying the bosonic commutation relations, $\left[\mathbf{f}(\mathbf{r}, \omega), \mathbf{f}^{\dagger}\left(\mathbf{r}^{\prime}, \omega^{\prime}\right)\right]=\delta\left(\mathbf{r}-\mathbf{r}^{\prime}\right) \delta\left(\omega-\omega^{\prime}\right)$ and $\left[\mathrm{f}(\mathbf{r}, \omega), \mathrm{f}\left(\mathbf{r}^{\prime}, \omega^{\prime}\right)\right]=0$. The electromagnetic Green's tensor (GT), $\overline{\overline{\mathbf{G}}}\left(\mathbf{r}, \mathbf{r}^{\prime}, \omega\right)$ describing the system response at $\mathbf{r}$ to a point source at $\mathbf{r}^{\prime}$, is determined by the Helmholtz equation:

$$
\left(\nabla \times \nabla \times-\frac{\omega^{2}}{c^{2}} \varepsilon(\mathbf{r}, \omega)\right) \cdot \overline{\overline{\mathbf{G}}}\left(\mathbf{r}, \mathbf{r}^{\prime}, \omega\right)=\delta\left(\mathbf{r}-\mathbf{r}^{\prime}\right) \overline{\overline{\mathbf{I}}},
$$

where $\overline{\overline{\mathbf{I}}}$ is the identity tensor.

The dynamics of the density operator $\rho$ for the TIA, after tracing out over the degrees of freedom of the field, can be described by a master equation [15]:

$$
\begin{aligned}
\frac{\partial \rho}{\partial t}= & -i \omega_{0} \sum_{j}\left[s_{j}^{z}, \rho\right]-i \sum_{i, j} \Omega_{i, j}\left[s_{i}^{+} s_{j}^{-}, \rho\right] \\
& -\sum_{i, j} \gamma_{i j}\left[s_{i}^{+} s_{j}^{-} \rho-2 s_{j}^{-} \rho s_{i}^{+}+\rho s_{i}^{+} s_{j}^{-}\right],
\end{aligned}
$$

where $\rho$ is the reduced density operator of the atomic system, $s_{j}^{+}=\left|e_{j}\right\rangle\left\langle g_{j}\right| \quad$ and $s_{j}^{-}=\left|g_{j}\right\rangle\left\langle e_{j}\right|$ are, respectively, the raising and lowering operators between the ground state $\left|g_{j}\right\rangle$ and the excited state $\left|e_{j}\right\rangle$ of the $j$ th atom, and $s_{j}^{z}=\frac{1}{2}\left(\left|e_{j}\right\rangle\left\langle e_{j}|-| g_{j}\right\rangle\left\langle g_{j}\right|\right)$. Here the parameter $\gamma_{i j}$ for $(i=j)$ describes the atomic spontaneous emission $j$ th atom, and for $(i \neq j)$ represents the collective damping rates of the TIA. This parameter is defined in terms of the electromagnetic GT of the system as follows [14]:

$$
\gamma_{i j}=\frac{2}{\hbar \varepsilon_{0}} \frac{\omega_{0}}{c^{2}} \operatorname{Im}\left[\mathrm{p}_{i} \cdot \overline{\overline{\mathrm{G}}}\left(\mathbf{r}_{\mathrm{i}}, \mathbf{r}_{\mathrm{j}}, \omega_{0}\right) \cdot \mathrm{p}_{j}^{*}\right],
$$

where $\mathrm{p}_{i}(i=1,2)$ is the dipole moment of the $i$ th atom, Im refers to the imaginary part of the function. In addition, the parameter $\Omega_{i j}$ is defined by: 


$$
\Omega_{i j}=-\frac{1}{\hbar \varepsilon_{0}} \frac{\omega_{0}}{c^{2}} \operatorname{Re}\left[\mathrm{p}_{i} \cdot \overline{\overline{\mathrm{G}}}\left(\mathbf{r}_{\mathrm{i}}, \mathbf{r}_{\mathrm{j}}, \omega_{0}\right) \cdot \mathrm{p}_{j}^{*}\right]
$$

where Re denotes the real part of the function. Here, the parameter $\Omega_{i j}$ for $(i=j)$ describes the Lamb shift of the $i$ th level of the TIA, and $(i \neq j)$ indicates the dipole-dipole interaction between TIA.

In order to calculate the degree of entanglement of the atomic system, we use the concurrence measure. This measure in terms of the eigenvalues of the matrix $\rho \tilde{\rho}$, that is $\lambda_{i}(i=1, \ldots, 4)$, is given by [16]:

$$
C=\max \left(0, \sqrt{\lambda_{1}}-\sqrt{\lambda_{2}}-\sqrt{\lambda_{3}}-\sqrt{\lambda_{4}}\right)
$$

where $\tilde{\rho}$ is defined as:

$$
\tilde{\rho}=\sigma_{y} \otimes \sigma_{y} \rho^{*} \sigma_{y} \otimes \sigma_{y} .
$$

Here, $\sigma_{y}$ represents the $y$ component of the Pauli matrix and $\rho^{*}$ denotes the complex conjugate of $\rho$.

By finding the solutions of Eq. (3) and substituting them into Eq. (6), the concurrence can be written as [14]:

$$
C(t)=e^{-\gamma_{s} t} \sqrt{\sinh ^{2}\left(\gamma_{c}\right)+\sin ^{2}\left(2 \Omega_{c} t\right)},
$$

where the collective parameters $\gamma_{s}, \gamma_{c}, \Omega_{s}$ and $\Omega_{c}$ are defined by:

$$
\begin{aligned}
& \gamma_{s}=\gamma_{11}=\gamma_{22}, \gamma_{c}=\gamma_{12}=\gamma_{21}, \\
& \Omega_{s}=\Omega_{11}=\Omega_{22}, \Omega_{c}=\Omega_{12}=\Omega_{21} .
\end{aligned}
$$

From the above equations, it is clear that all information we need to calculate the concurrence is contained in the electromagnetic GT. Therefore, we must first calculate the GT of the system. By using the scattering superposition method, the EGT can be written as [17]:

$$
\overline{\overline{\mathbf{G}}}^{(f s)}\left(\mathbf{r}, \mathbf{r}^{\prime}\right)=\overline{\overline{\mathbf{G}}}_{o}\left(\mathbf{r}, \mathbf{r}^{\prime}\right) \delta_{f}^{s}+\overline{\overline{\mathbf{G}}}_{S}^{(f s)}\left(\mathbf{r}, \mathbf{r}^{\prime}\right) .
$$

Here, the superscript $(f s)$ denotes the layers where the field point and source point locate, the vacuum GT, $\overline{\overline{\mathbf{G}}}_{o}\left(\mathbf{r}, \mathbf{r}^{\prime}\right)$, corresponds to the contribution from the source in the infinite vacuum space while the scattering GF, $\overline{\overline{\mathbf{G}}}_{S}^{(f s)}\left(\mathbf{r}, \mathbf{r}^{\prime}\right)$, reflects the contribution of the source due to the presence of the nanosphere interface. When the field and source points are located out of the SNS, these two contributions in the spherical coordinate system can be expressed as follows:

$$
\begin{aligned}
\overline{\overline{\mathrm{G}}}_{0}\left(\mathrm{r}, \mathrm{r}^{\prime}\right)= & \frac{\hat{r} \hat{r}}{k_{1}^{2}} \delta\left(r-r^{\prime}\right)+\frac{i k_{s}}{4 \pi} \sum_{n=0}^{\infty} \sum_{m=0}^{n}\left(2-\delta_{m}^{0}\right) \frac{2 n+1}{n(n+1)} \frac{(n-m) !}{(n+m) !} \times \\
& \times\left(\mathrm{M}_{m n}^{(1)}\left(k_{1}\right) \mathrm{M}_{m n}^{\prime}\left(k_{1}\right)+\mathrm{N}_{m n}^{(1)}\left(k_{1}\right) \mathrm{N}_{m n}^{\prime}\left(k_{1}\right)\right),
\end{aligned}
$$

$$
\begin{aligned}
& \overline{\overline{\mathrm{G}}}_{S}^{(11)}\left(\mathrm{r}, \mathrm{r}^{\prime}\right)=\frac{i k_{1}}{4 \pi} \sum_{n=0}^{\infty} \sum_{m=0}^{n}\left(2-\delta_{m}^{0}\right) \frac{2 n+1}{n(n+1)} \frac{(n-m) !}{(n+m) !} \\
& \times\left(B_{M}{ }^{11} \mathrm{M}_{m n}^{(1)}\left(k_{1}\right) \mathrm{M}_{m n}^{(1)}\left(k_{1}\right)+B_{N}{ }^{11} \mathrm{~N}_{m n}^{(1)}\left(k_{1}\right) \mathrm{N}_{m n}^{\prime(1)}\left(k_{1}\right)\right) .
\end{aligned}
$$

Here, the prime (non-prime) denotes the coordinates of the source (field) point, $\mathbf{M}$ and $\mathbf{N}$ are the spherical vector wave functions defined as [17]:

$$
\begin{aligned}
\mathbf{M}_{o m n}^{(l)}\left(k_{1}\right)= & \mp \frac{m}{\sin \theta} z_{n}^{(l)}\left(k_{1} r\right) P_{n}^{m}(\cos \theta){ }_{\cos }^{\sin } m \phi \hat{\theta} \\
& -z_{n}^{(l)}\left(k_{1} r\right) \frac{d P_{n}^{m}(\cos \theta)}{d \theta}{ }_{\sin }^{\cos } m \phi \hat{\phi},
\end{aligned}
$$

$$
\begin{aligned}
\mathbf{N}_{\delta^{\prime} m m}^{(l)}\left(k_{1}\right)= & \frac{n(n+1)}{k_{1} r} z_{n}^{(l)}\left(k_{1} r\right) P_{n}^{m}(\cos \theta) \underset{\sin }{\cos } m \phi \hat{r} \\
& +\frac{1}{k_{1} r} \frac{d\left[r z_{n}^{(l)}\left(k_{1} r\right)\right]}{d r} \\
& \times\left[\frac{d P_{n}^{m}(\cos \theta)}{d \theta} \sin _{\sin }^{\cos } m \phi \hat{\theta} \mp \frac{m}{\sin \theta} P_{n}^{m}(\cos \theta)_{\cos }^{\sin } m \phi \hat{\phi}\right] .
\end{aligned}
$$


where $k_{1}=\omega / c$, and $z_{n}^{(l)}\left(k_{1} r\right)$ for $l=0$ and $l=1$ indicate the first type spherical Bessel function, $J_{n}\left(k_{1} r\right)$, and the third-type spherical Bessel function or the first-type spherical Hankel function, $h_{n}^{(1)}\left(k_{1} r\right)$, respectively. By imposing the boundary conditions on the GT at the surface of the SNS, the unknown coefficients $B_{M, N}^{11}$ are given by:

$$
B_{M, N}^{11}=-R_{F, 1}^{H, V}
$$

where the subscript $F$ refers to the centrifugal wave. Furthermore, the superscripts of the TE and TM waves are represented by the $H$ and $V$, respectively. Here, the equivalent reflection coefficient $R_{F, 1}^{H, V}$ is defined as:

$$
\begin{aligned}
& R_{F 1}^{H}=\frac{\mu_{1} k_{2} \partial \varsigma_{21} \varsigma_{11}-\mu_{2} k_{1} \partial \varsigma_{11} \varsigma_{21}}{\mu_{1} k_{2} \partial \varsigma_{21} \hbar_{11}-\mu_{2} k_{1} \varsigma_{21} \partial \hbar_{11}}, \\
& R_{F 1}^{V}=\frac{\mu_{1} k_{2} \varsigma_{21} \partial \varsigma_{11}-\mu_{2} k_{1} \varsigma_{11} \partial \varsigma_{21}}{\mu_{1} k_{2} \varsigma_{21} \partial \hbar_{11}-\mu_{2} k_{1} \partial \varsigma_{21} \hbar_{11}},
\end{aligned}
$$

where

$$
\begin{aligned}
& \varsigma_{i l}=j_{n}\left(k_{i} a_{l}\right), \\
& \hbar_{i l}=h_{n}^{(1)}\left(k_{i} a_{l}\right), \\
& \partial \varsigma_{i l}=\left.\frac{1}{\rho} \frac{d\left[\rho j_{n}(\rho)\right]}{d \rho}\right|_{\rho=k_{i} a_{l}}, \\
& \partial \hbar_{i l}=\left.\frac{1}{\rho} \frac{d\left[\rho h_{n}^{(1)}(\rho)\right]}{d \rho}\right|_{\rho=k_{i} a_{l}} .
\end{aligned}
$$

Now, we can use equation (8) to the TIA system where their atomic dipole moment may be perpendicular to the interface along the $\mathrm{z}$ axis and/or parallel to the interface along the $x$ axis, with the GT obtained in Eqs. (9)-(11) and making use of the symmetry of our system, after some algebra and manipulations, the collective parameters for the two special cases of radial and tangential directions can be written as:

$$
\begin{aligned}
& \gamma_{11, r r}=\frac{6 \pi}{\omega} \operatorname{Im}\left[\frac { i k _ { 1 } } { 4 \pi } \sum _ { n = 0 } ^ { \infty } \frac { ( 2 n + 1 ) n ( n + 1 ) } { k _ { 1 } ^ { 2 } r ^ { 2 } } \left\{h_{n}^{(1)}\left(k_{1} r\right) J_{n}\left(k_{1} r\right)+\right.\right. \\
& \left.\left.B_{N}^{11}\left(h_{n}^{(1)}\left(k_{1} r\right)\right)^{2}\right\}\right] \text {, } \\
& \gamma_{12, r r}=\frac{6 \pi}{\omega} \operatorname{Im}\left[\frac { i k _ { 1 } } { 4 \pi } \sum _ { n = 0 } ^ { \infty } \frac { ( 2 n + 1 ) n ( n + 1 ) } { k _ { 1 } ^ { 2 } r ^ { 2 } } ( - 1 ) ^ { n } \left\{h_{n}^{(1)}\left(k_{1} r\right) J_{n}\left(k_{1} r\right)\right.\right. \\
& \left.\left.+B_{N}^{11}\left(h_{n}^{(1)}\left(k_{1} r\right)\right)^{2}\right\}\right] \text {, } \\
& \Omega_{12, r r}=-\frac{3 \pi}{\omega} \operatorname{Re}\left[\frac { i k _ { 1 } } { 4 \pi } \sum _ { n = 0 } ^ { \infty } \frac { ( 2 n + 1 ) n ( n + 1 ) } { ( k _ { 1 } r ) ^ { 2 } } ( - 1 ) ^ { n } \left\{h_{n}^{(\mathrm{l})}\left(k_{1} r\right) J_{n}\left(k_{1} r\right)\right.\right. \\
& \left.\left.+B_{N}^{11}\left(h_{n}^{(1)}\left(k_{1} r\right)\right)^{2}\right\}\right] \text {. } \\
& \gamma_{11, \theta \theta}=\frac{6 \pi}{\omega} \operatorname{Im}\left[\frac{i k_{1}}{4 \pi} \sum_{n=0}^{\infty} \frac{(2 n+1)}{2}\right. \\
& \times\left\{h_{n}^{(1)}\left(k_{1} r\right) J\left(k_{1} r\right)+\partial h_{n}^{(1)}\left(k_{1} r\right) \partial J_{n}\left(k_{1} r\right)\right. \\
& \left.\left.+B_{M}^{11}\left(h_{n}^{(1)}\left(k_{1} r\right)\right)^{2}+B_{N}^{11}\left(\partial h_{n}^{(1)}\left(k_{1} r\right)\right)^{2}\right\}\right] \text {, } \\
& \gamma_{12, \theta \theta}=\frac{6 \pi}{\omega} \operatorname{Im}\left[\frac{i k_{1}}{4 \pi} \sum_{n=0}^{\infty} \frac{(2 n+1)}{2}(-1)^{n+1}\right. \\
& \times\left\{h_{n}^{(1)}\left(k_{1} r\right) J_{n}\left(k_{1} r\right)+\partial h_{n}^{(1)}\left(k_{1} r\right) \partial J_{n}\left(k_{1} r\right)\right. \\
& \left.\left.+B_{M}^{11}\left(h_{n}^{(1)}\left(k_{1} r\right)\right)^{2}+B_{N}^{11}\left(\partial h_{n}^{(1)}\left(k_{1} r\right)\right)^{2}\right\}\right] \text {, } \\
& \Omega_{12, \theta \theta}=-\frac{3 \pi}{\omega} \operatorname{Re}\left[\frac{i k_{1}}{4 \pi} \sum_{n=0}^{\infty} \frac{(2 n+1)}{2}(-1)^{n+1}\right. \\
& \times\left\{h_{n}^{(1)}\left(k_{1} r\right) J_{n}\left(k_{1} r\right)+\partial h_{n}^{(1)}\left(k_{1} r\right) \partial J_{n}\left(k_{1} r\right)\right. \\
& \left.\left.+B_{M}^{11}\left(h_{n}^{(1)}\left(k_{1} r\right)\right)^{2}+B_{N}^{11}\left(\partial h_{n}^{(1)}\left(k_{1} r\right)\right)^{2}\right\}\right] \text {, } \\
& \text { where } \quad \partial h_{n}^{(1)}(\rho)=\frac{1}{\rho} \frac{d\left[\rho h_{n}^{(1)}(\rho)\right]}{d \rho} \quad \text { and } \\
& \partial J_{n}(\rho)=\frac{1}{\rho} \frac{d\left[\rho J_{n}(\rho)\right]}{d \rho} .
\end{aligned}
$$

Finally, by inserting the above equations in Eq. (7), the concurrence of the atomic system is obtained.

\section{III.NUMERICAL RESULTS AND}

The complexity of the calculated equation for the concurrence makes it difficult to predict the results analytically. Therefore, we perform the numerical calculations for the collective decay rate (8) and the concurrence (7). To do 
this, we characterize the permittivity function of the SNS by the Drude-Lorentz model: $\varepsilon_{m}=\varepsilon_{\infty}-\omega_{p}^{2} /\left(\omega^{2}-i \gamma \omega\right)$, where $\varepsilon_{\infty}$ is the static permittivity, $\omega_{p}$ is the plasma frequency and $\gamma$ is the damping coefficient of the SNS. Here, we take the typical parameters $\varepsilon_{\infty}=6, \omega_{p}=7.90 \mathrm{ev}$ and $\gamma=51 \mathrm{mev}$ for the SNS [18]. For our numerical calculations, we assume a SNS with radius $a=20 \mathrm{~nm}$ and place the TIA at $r=22 \mathrm{~nm}$, that is $2 \mathrm{~nm}$ above the surface of the SNS. These material parameters imply a surface plasmonpolariton resonance $\omega_{s}=7.90 / \sqrt{1+\varepsilon_{\infty}} e V=2.97 \mathrm{eV}$.

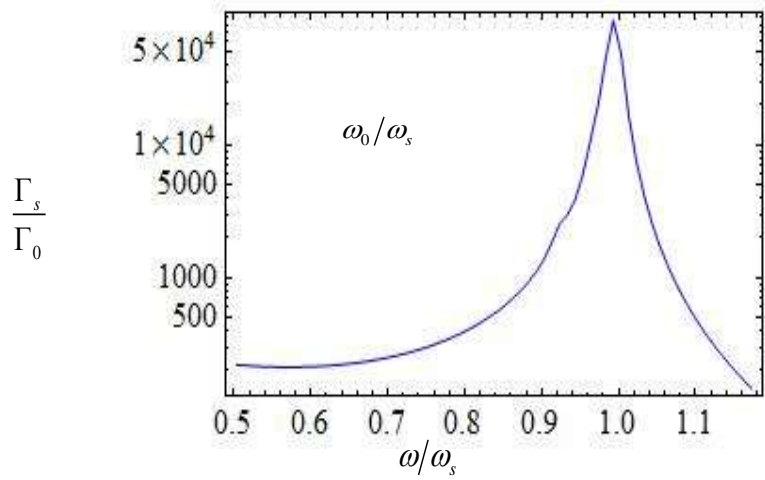

Fig. 1. The dimensionless spontaneous emission rate $\Gamma_{s} / \Gamma_{0}$ as a function of a dimensionless parameter $\omega_{0} / \omega_{s}$. Here, $\Gamma_{0}$ is the spontaneous emission rate of a single atom in the vacuum.

In Fig. 1, the spontaneous emission rate of a single excited atom near the SNS is plotted. As it is seen, the decay rate $\Gamma_{s} / \Gamma_{0}$ is dramatically increased at the frequency $\omega_{0}=\omega_{s}$. As it is known, the decay rate of atoms strongly depends on their local environment. In other words, the decay rate is proportional to the electromagnetic LDOS. Therefore, due to exciting LSP in the SNS, the LDOS is severely enhanced. In this manner, we observe a peak in the decay rate of the TIA at the excitation frequency of the LSP.

In Fig. 2, the time evolution of the concurrence (7) as function of the dimensionless time $\Gamma_{0} t$ is plotted for two different cases that atomic dipole moments are perpendicular (right panel) and parallel (left panel) to the SNS, respectively. The blue solid and the red dotdashed lines represent the concurrence of the TIA when two atoms are at resonance with the LSP frequency $\omega_{0}=\omega_{s}$ and far from the LSP frequency, $\omega_{0}=0.1 \omega_{s}$, respectively. In all curves, the concurrence is zero at the initial time $t=0$. This is as it should be, because the TIA is initially prepared in a separable state $\left|e_{1}\right\rangle\left|g_{2}\right\rangle$. As time progresses, in the case that two atoms are at resonance with the SNS, the concurrence is nearly zero. This is a result of the fact that LSP resonance frequency for the SNS occur at frequency where the absorption is so strong so that the emitted photon by the excited atom is absorbed via the SNS. Therefore, there is no entanglement between the TIA.
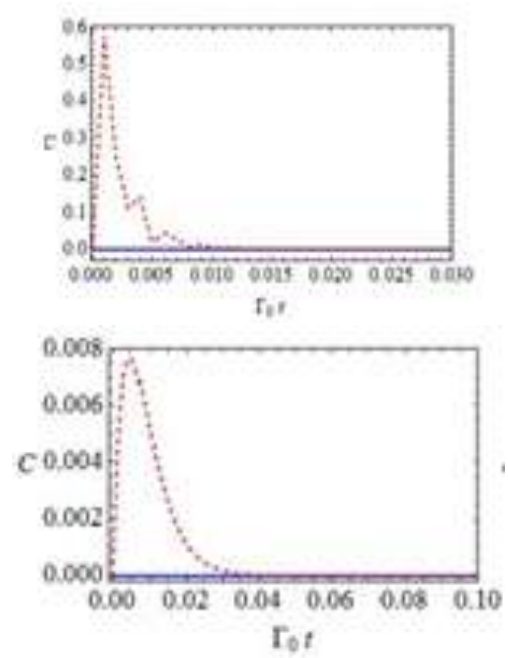

Fig. 2. The time evolution of the concurrence $\mathrm{C}$ as a function of a dimensionless parameter $\Gamma_{0} t$. The top and bottom panels correspond to the case that the atomic dipole moment are perpendicular and parallel to the surface of the SNS, respectively.

When the transition frequency of the TIA is far from the excitation frequency of the LSP, the concurrence is characterized by a fast initial increase followed by a very slow decay. This dynamics can be easily understood from the time evolution of the populations of the collective symmetric and antisymmetric states $| \pm\rangle=\left(\left|e_{1}\right\rangle\left|g_{2}\right\rangle \pm\left|g_{1}\right\rangle\left|e_{2}\right\rangle\right) / \sqrt{2}[14]$ 
In contrast to the SNS, LSP resonance frequencies for a nanoshell depend significantly on the shell thickness. Therefore, the LSP resonances occur at lower frequencies where the absorption inside the nanoshell is weak. Therefore, we expect that the entanglement between two atoms can be mediated by phonons through the nanoshell, which is under construction.

\section{CONCLUSION}

We have studied the entanglement of two-level atoms near a spherical silver nanoparticle. By using the concurrence measure, the entanglement dynamics of the aforementioned atomic system is investigated. The numerical results show significant enhancement in the decay rates near the excitation frequency of the localized plasmonpolariton, while the concurrence is almost zero.

\section{REFERENCES}

[1] C.H. Bennett and S.J. Wiesner, "Communication via one- and two-particle operators on Einstein-Podolsky-Rosen states," Phys. Rev. Lett. Vol. 69, pp 2881-2884, 1992.

[2] E. Boukobza and D. Tannor, "Entropy exchange and entanglement in the JaynesCummings model," Phys. Rev. A, Vol. 71, pp. 063821 (1-8), 2005.

[3] W.K. Wootters, "Entanglement of Formation of an Arbitrary State of Two Qubits," Phys. Rev. Lett. Vol. 80, pp. 2245-2248, 1998.

[4] B. Huttner and S.M. Barnett, "Quantization of the electromagnetic field in dielectrics," Phys. Rev. A, Vol. 46, pp 4306-4322, 1992.

[5] J. Jeffers, S.M. Barnett, R. Loudon, R. Matloob, and M. Artoni, "Canonical quantum theory of light propagation in amplifying media," Opt. Commun. Vol. 131, pp. 66-71, 1996.

[6] L.G. Suttorp and M. Wubs, "Field quantization in inhomogeneous absorptive dielectrics," Phys. Rev. A, Vol. 70, pp. 013816 (1-18), 2004.

[7] M. Amooshahi, "Canonical quantization of electromagnetic field in an anisotropic polarizable and magnetizable medium," J. Math. Phys. Vol. 50, pp. 062301(1-22), 2009.

[8] F. Kheirandish and E. Amooghorban, "Finitetemperature Cherenkov radiation in the presence of a magnetodielectric medium," Phys. Rev. A, Vol. 82, pp. 042901(1-14), 2010.

[9] F. Kheirandish, E. Amooghorban, and M. Soltani, "Finite-temperature Casimir effect in the presence of nonlinear dielectrics," Phys. Rev. A, Vol. 83, pp. 032507 (1-10), 2011.

[10]E. Amooghorban, M. Wubs, N.A. Mortensen, and F. Kheirandish, "Casimir forces in multilayer magnetodielectrics with both gain and loss," Phys. Rev. A, Vol. 84, pp. 013806 (1-15), 2011.

[11]R. Matloob, R. Loudon, S.M. Barnett, and J. Jeffers, "Electromagnetic field quantization in absorbing dielectrics," Phys. Rev. A, Vol. 52, pp. 4823-4838, 1995.

[12]L. Knöll, S. Scheel, and D.-G.Welsch, Coherence and Statistics of Photons and Atoms, Wiley New York, 2001.

[13]R. Matloob, "Electromagnetic field quantization in a linear isotropic permeable dielectric medium," Phys. Rev. A, Vol. 70, pp. 022108 (1-9), 2004.

[14]E. Amooghorban and E. Aleebrahim, "Entanglement dynamics of two two-level atoms in the vicinity of an invisibility cloak," Phys. Rev. A, Vol. 96, pp. 012339 (1-11) 2017.

[15]S.A. Biehs and G.S. Agarwal, "Qubit entanglement across $\varepsilon$-near-zero media," Phys. Rev. A, Vol. 96, pp. 022308 (1-9), 2017.

[16]Z. Ficek, R. Tanas, and S.Kielich, "Quantum beats and superradiant effects in the spontaneous emission from two nonidentical atoms," Physica. A, Vol. 146, pp. 452-482 1987.

[17]L. Li, P. Kooi, M. Leong, and T. Yeo, "Electromagnetic dyadic Green's function in spherically multilayered media," IEEE Trans. Microwave Theory Tech. Vol. 42, pp. 23022310, 1994.

[18]C. van Vlack and P. Hughes, "Spontaneous emission spectra and quantum light-matter interactions from a strongly coupled quantum 
dot metal-nanoparticle system," Phys. Rev. B, Vol. 85, pp. 075303 (227-241), 2012.

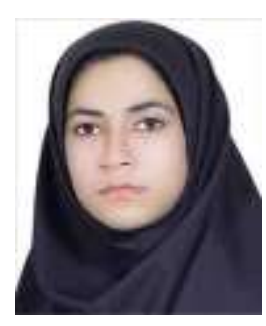

Roya Mombeiny Godazhdar was born in Ahwaz, Iran. She received the B.Sc. degree from Ahwaz Payame Noor University, Iran in 2017. She is currently pursuing the M.Sc degree in Shahrekord University. Her research interests include Quantum optics.

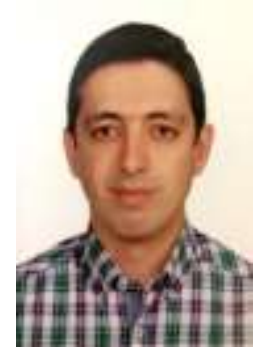

Ehsan Amooghorban was born in Isfahan, Iran. He received the B.Sc., M.Sc. and Ph.D. degrees in atomic and molecular physics from the University of Isfahan, Iran, in 2004, 2006 and 2011, respectively. He passed his research period as a visitor in the photonic department of the Technical University of Denmark
(DTU) from 2010 to 2011 . He is currently an associate professor at the physics department and a member of photonics research group and nanotechnology research center at Shahrekord University, Iran. His research interests include quantum optics, metamaterial and theoretical foundations of quantum mechanics.

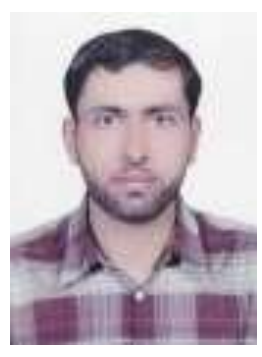

Ali Mahdifar was born in Isfahan, Iran, on 1977. He received the B.Sc. and M.Sc. degrees in Condensed Matter Physics from Isfahan University of Technology and Ph.D degree in Atomic and Molecular Physics from the Isfahan University, respectively, in 2000, 2003 and 2007. He started his career at the physics department of Shahrekord University as assistant professor in 2007. He later (in 2018) joined the University of Isfahan, where he is presently an Associate Professor. He is also a member of Isfahan Quantum Optics Group. His research interests include geometrical structure of coherent states and their applications, theoretical quantum optics and mathematical (geometrical) and theoretical foundations of quantum mechanics. 
THIS PAGE IS INTENTIONALLY LEFT BLANK. 